\title{
ChemPlot, a Python library for chemical space visualization
}

\author{
Murat Cihan Sorkun ${ }^{1,2,3}$, Dajt Mullaj ${ }^{1,2}$, J. M. Vianney A. Koelman ${ }^{1,2,3}$, and Süleyman Er ${ }^{1,2 *}$
}

Visualizing chemical spaces streamlines the analysis of molecular datasets by reducing the information to human perception level, hence it forms an integral piece of molecular engineering, including chemical library design, highthroughput screening, diversity analysis, and outlier detection. We present here ChemPlot, which enables users to visualize the chemical space of molecular datasets in both static and interactive ways. ChemPlot features structural and tailored similarity methods, together with three different dimensionality reduction methods: PCA, t-SNE, and UMAP. ChemPlot is the first visualization software that tackles the activity/property cliff problem by incorporating tailored similarity. With tailored similarity, the chemical space is constructed in a supervised manner considering target properties. Additionally, we propose a metric, the Distance Property Relationship score, to quantify the property difference of similar (i.e. close) molecules in the visualized chemical space. ChemPlot can be installed via Conda or PyPi (pip). Its web application is freely accessible at https://www.amdlab.nl/chemplot/.

\section{INTRODUCTION}

Data visualization is an essential tool for scientists to reveal patterns that are hidden in high-dimensional data, to interpret these patterns, and - as a matter of usual practice - to convey them to non-experts. Moreover, in the current era of artificial intelligence (AI)-guided chemical design, visualization is frequently employed to describe the applicability domains of data-driven models. In molecular data science, the complex variability of molecular data, as stored in high-dimensional spaces spanned by graphs, textual representation, atom coordinates, or any combinations thereof, demands the development of chemically informative data visualization methods and tools. The data visualization provides a practical means to reduce the high-dimensional molecular data to spaces of two (2D) or three dimensions (3D). A visual inspection of low-dimensional chemical space data enables a more realistic screening of molecules with user-desired features, in addition to exploratory analysis of the data, including but not limited to, diversity and outlier detection.

Chemical space is a multi-dimensional space in which the alike molecules are grouped together based on their similarities. The chemical space terminology is commonly used in two distinct, yet related, concepts. First, it denotes the property space of an unknown size, which is spanned by all molecules that exist or are likely to exist. Secondly, it also finds use in the context of a given molecular dataset with a finite size. In the current study, we refer to the latter terminology of chemical space and focus on quantifying the similarities of molecules within domain-specific datasets.

\footnotetext{
${ }^{1}$ DIFFER - Dutch Institute for Fundamental Energy Research, De Zaale 20, Eindhoven 5612 AJ, the Netherlands.

${ }^{2}$ CCER - Center for Computational Energy Research, De Zaale 20, Eindhoven 5612 AJ, the Netherlands.

${ }^{3}$ Department of Applied Physics, Eindhoven University of Technology, Eindhoven 5600 MB, the Netherlands. *email: s.er@differ.nl
} 
Visualization of chemical spaces usually consists of three main steps. In the first step, molecular representations (e.g. SMILES or InChI) are converted into binary or real-value arrays. Each element of the array corresponds to a dimension in the chemical space. In the second step, the high-dimensional chemical space is reduced to $2 \mathrm{D}$ or $3 \mathrm{D}$ spaces. In the final step, the reduced chemical space is converted into a visual representation. Fingerprints and descriptors ${ }^{1-3}$ are the most widely used molecular encoding methods for the first step. Principal component analysis (PCA) ${ }^{4}$, self-organized maps $(\mathrm{SOM})^{5}$, multidimensional scaling (MDS) ${ }^{6}$, and t-distributed stochastic neighbor embedding (t-SNE) ${ }^{7}$ are the commonly used dimensionality reduction methods. Finally, visualizations can take the shape of either 2D or 3D, static or interactive, scatter or grid-like plots. In addition to these, there are alternative chemical space visualization approaches including the minimum spanning-trees ${ }^{8}$, similarity networks ${ }^{9}$, and uniform manifold approximation and projection (UMAP) ${ }^{10}$.

In practice, screening of molecules with desired properties is usually based on the similarity property principle (SPP), which assumes that similar molecules (close to each other in chemical space) show similar properties. However, it is not uncommon for pairs of similar molecules to violate the SPP principle ${ }^{11}$ and such violations are referred to as activity/property cliffs (APCs). APCs may mislead the screening and result in a deceleration of the discovery process of molecules with target properties. It is important to spot or, even better, prevent APCs to facilitate a speedy discovery process. Several visualization tools include additional analysis modules for APCs ${ }^{12-14}$. However, currently, there is no publicly available chemical space visualization tool that provides a remedy to the APC problem as part of the process.

Even though the majority of the chemical space visualization tools are distributed as an integrated feature of commercial software packages, several dedicated tools are also freely available ${ }^{15}$. In support of open science, the latter tools have been widely serving the chemical informatics community, although admitting that they carry the non-negligible sustainability concerns. For instance, free visualization tools are available either as standalone software ${ }^{12,13,16,17}$ or as web server applications ${ }^{14,18,19}$, however, they all require developer-dependent maintenance for version updates, bug-fixing, and adding new features. Therefore, in relation to the completion of code development projects, once effective tools may become inaccessible, outmoded, or face compliance issues with the continuously evolving operating systems.

Here, we present ChemPlot, a Python library for chemical space visualization. ChemPlot is free and its source code is available on GitHub under the Berkeley Source Distribution (BSD) license. ChemPlot is a user-friendly software, in which users can transform their datasets into interactive 2D chemical space visualizations. The current version(1.1.1) of ChemPlot provides three dimensionality reduction methods: PCA, t-SNE, and UMAP. Additionally, two similarity methods are supported: structural and tailored. According to our knowledge, ChemPlot is the first tool that applies tailored similarity ${ }^{20}$, which measures the similarity of molecules in a supervised manner considering the target properties of compounds, thus tackling the APC problem. Additionally, we propose a metric, the Distance Property Relationship score, to quantify the target property difference between molecules close to each other in chemical space. ChemPlot can be installed via Conda or PyPi (pip). Finally, alongside the user manual that contains detailed information and practical examples, we also provide a web interface to encourage the use of ChemPlot without the need for coding expertise (see Code Availability). 


\section{RESULTS}

In this section, we present the features of ChemPlot through the use of example codes and figures. We start with the installation of ChemPlot, continue with some examples of visualizations, and show its advanced functionalities. We introduce the available dimension reduction and similarity methods and compare their visualization performances. Lastly, we present the ChemPlot web application.

\section{Installation}

ChemPlot is an open-source Python library with its source code stored on a GitHub repository (see Code Availability). In addition to a direct installation from the repository, users can also install the deployed packages via Conda and PyPi package management environments. ChemPlot runs on the Python framework and it can be installed from two alternative sources via the command prompt using either of the following commands.

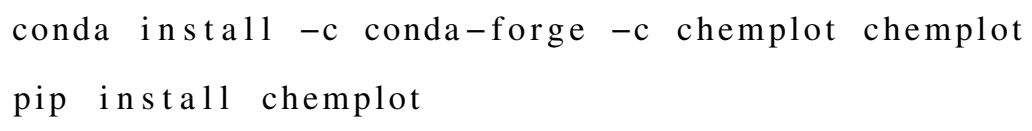

\section{Visualization}

To visualize the chemical space of their datasets, users first need to construct a Plotter object. The Plotter object can be created from a molecular dataset that includes either of the SMILES or InChI notations of compounds. The below code shows a basic t-SNE plotting example from the SMILES data.

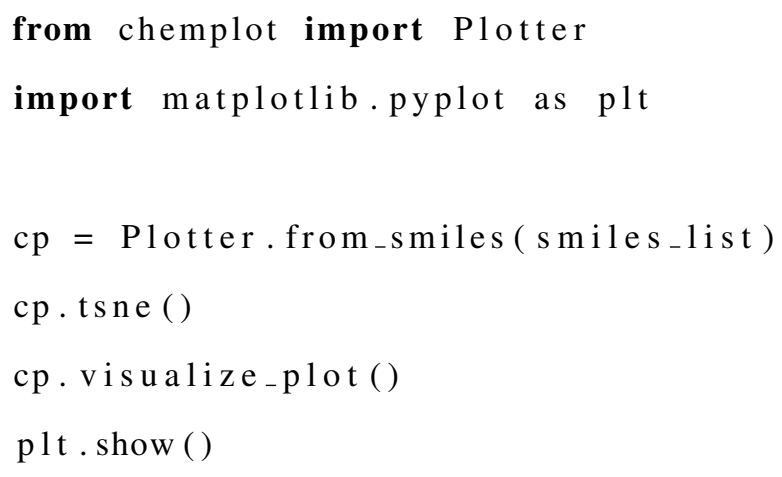

Figure 1a and 1c show the example visualizations of the chemical space of $\beta-$ secretase 1 (BACE) ${ }^{21}$ and Lipophilicity ${ }^{22}$ datasets using t-SNE with the default parameters.

\section{Target Coloring}

ChemPlot allows coloring the molecules based on a given target property. The given property is automatically classified as a numerical or categorical variable and the coloring is applied accordingly. Alternatively, users can also specify the target type themselves. As an illustration, the following code shows how the target assignment can be done while creating the Plotter object.

$$
c p=\text { Plotter.from_smiles(smiles_list, target=target_list, target_type="C") }
$$




\section{(a) BACE by t-SNE}

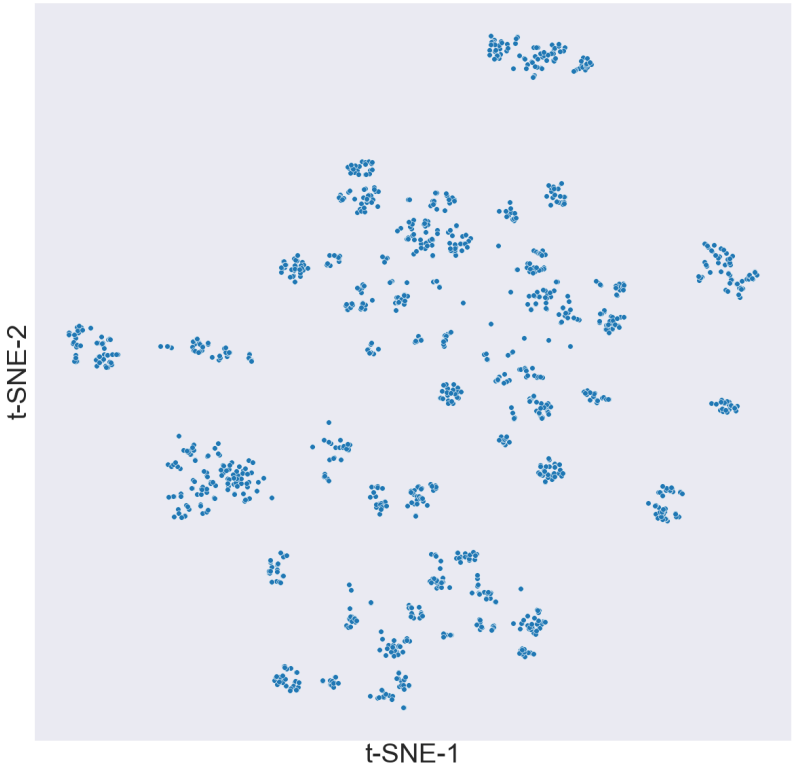

(c) Lipophilicity by t-SNE

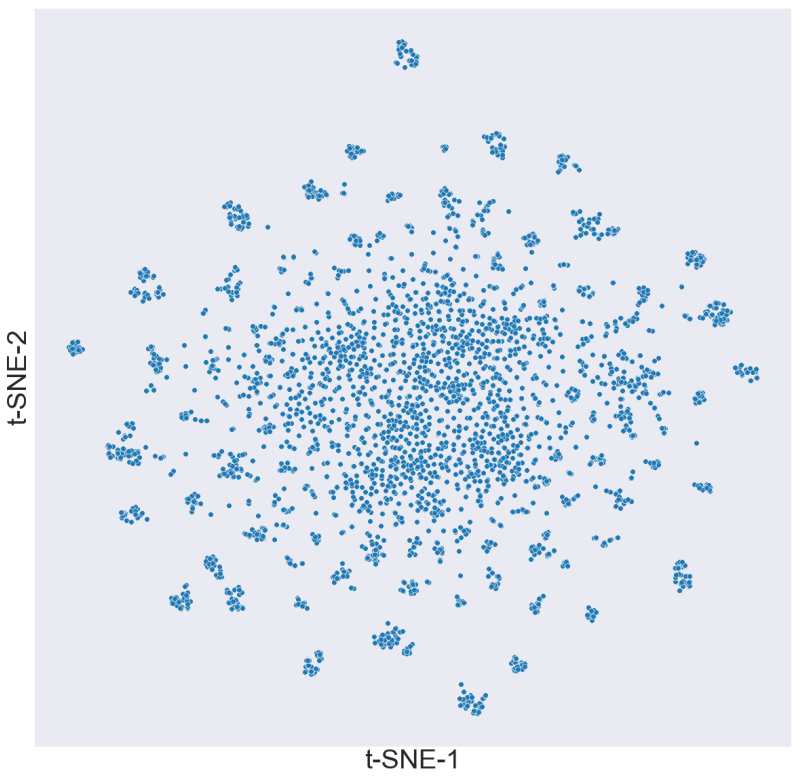

(b) BACE by t-SNE (target colored)

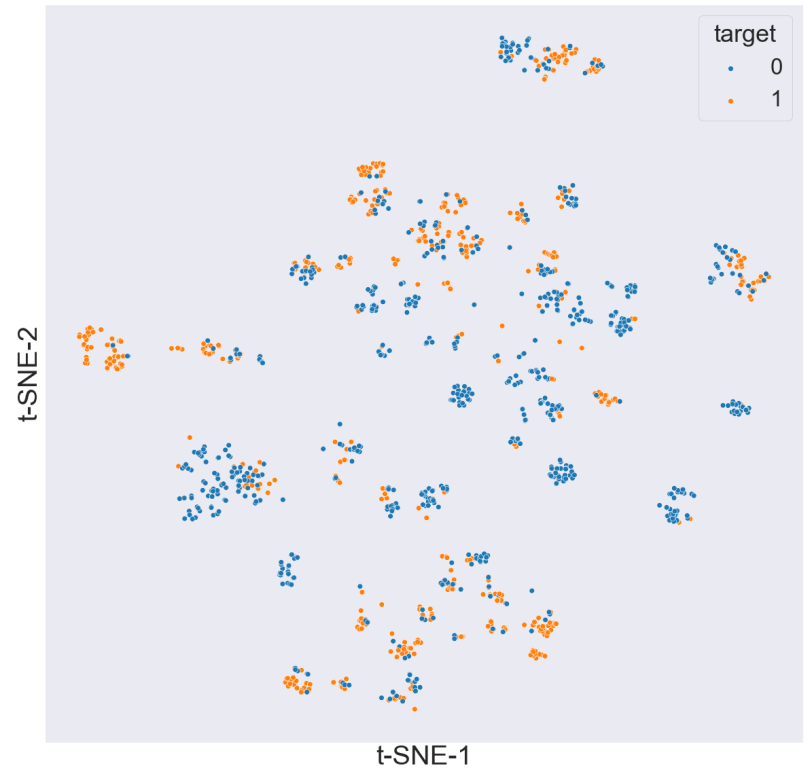

(d) Lipophilicity by t-SNE (target colored)

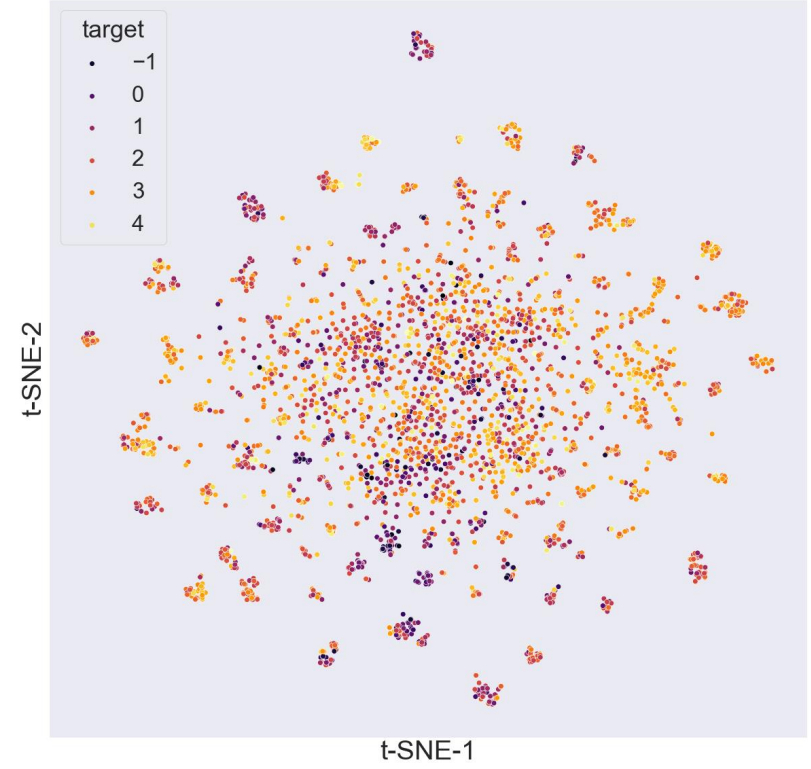

Figure 1. BACE (a and $\mathbf{b}$ ) and Lipophilicity (c and $\mathbf{d}$ ) datasets visualized by using ChemPlot. In $\mathbf{a}$ and $\mathbf{c}$, all the data points are shown with a single color, whereas in $\mathbf{b}$ and $\mathbf{d}$, they are colored according to the targets' values. In $\mathbf{b}$, the target properties are categorical values and therefore the points are colored according to the category of the target. In $\mathbf{d}$, the target properties are continuous values and therefore the points are colored according to the corresponding color from the predefined colormap. The corresponding values for the class colors and the gradient intervals are shown in the legends.

Figure $1 \mathrm{~b}$ and $1 \mathrm{~d}$ show the example visualizations of the chemical space of BACE (categorical) and Lipophilicity (numerical) dataset using t-SNE and with colors according to the targets. 
(a) BBBP by PCA

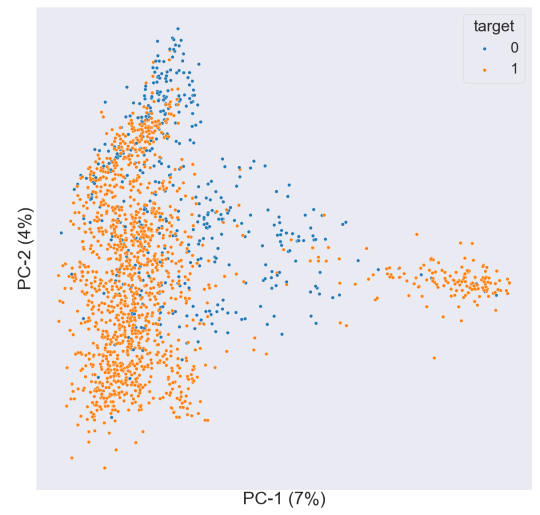

(d) AqSolDB by PCA

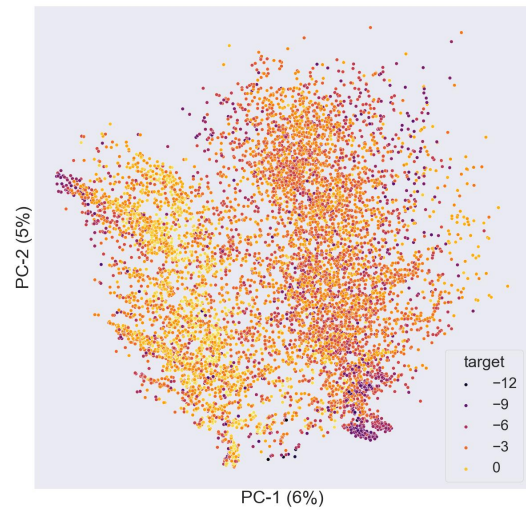

(b) BBBP by t-SNE

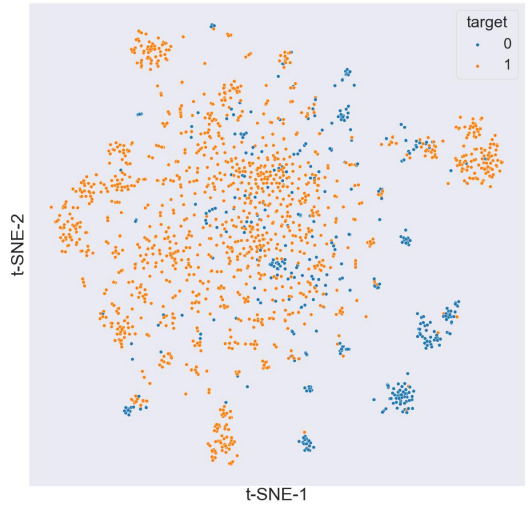

(e) AqSolDB by t-SNE

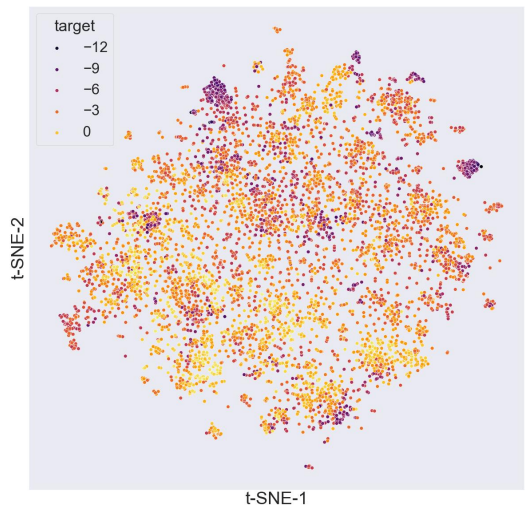

(c) BBBP by UMAP

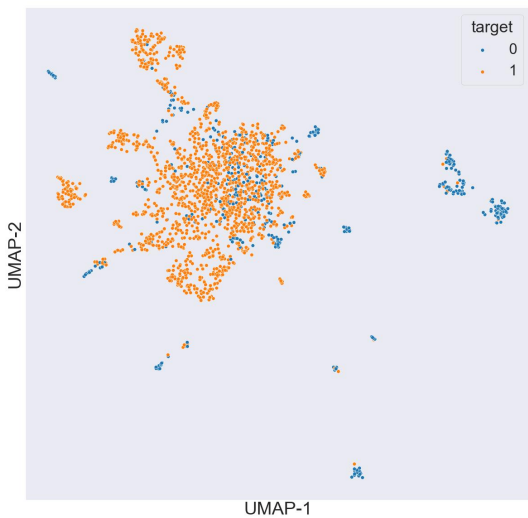

(f) AqSolDB by UMAP

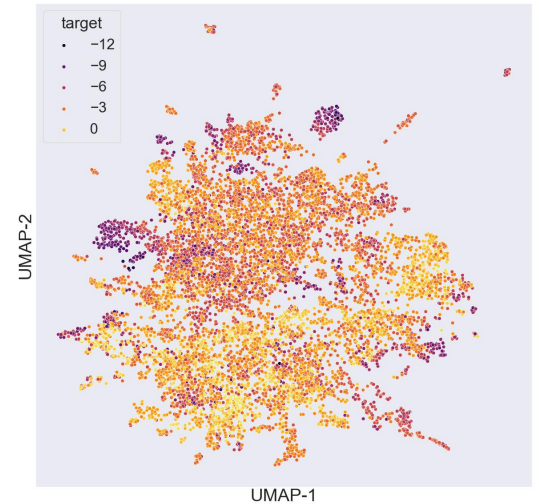

Figure 2. $\mathrm{BBBP}(\mathbf{a}, \mathbf{b}$, and $\mathbf{c})$ and $\mathrm{AqSolDB}(\mathbf{d}, \mathbf{e}$, and $\mathbf{f})$ datasets visualized by using structural similarity. In $\mathbf{a}$ and $\mathbf{d}$, the dimensions are reduced by PCA. The first and the second principal components are used as the horizontal and vertical axes, respectively. The explained variances of each component are given in parenthesis. In $\mathbf{b}$ and $\mathbf{e}$, the dimensions are reduced by t-SNE. In $\mathbf{c}$ and $\mathbf{d}$, the dimensions are reduced by UMAP.

\section{Dimensionality Reduction}

ChemPlot implements three different dimensionality reduction methods (PCA, t-SNE, and UMAP) to map the molecules onto 2D. Example visualizations of Blood-brain barrier penetration (BBBP $)^{23}$ and $\mathrm{AqSolDB}^{24}$ datasets using these algorithms are shown in Figure 2. While PCA provides a linear projection of given dimensions, both t-SNE and UMAP apply non-linear 2D mappings by clustering and locating molecules depending on their local neighborhoods. Users can assign parameters for t-SNE and UMAP, or alternatively, they can directly use the optimized values that are calculated and assigned automatically by ChemPlot. Additional details on the technicalities of dimensionality reduction algorithms are provided in the Methods section. The following code shows the execution of three different dimensionality reduction methods that are currently available in ChemPlot.

$$
\text { cp.pca() // cp.tsne() // cp.umap() }
$$

\section{Molecular Similarity}

ChemPlot implements two types, structural and tailored, of molecular similarity methods. Structural similarity uses only the 
generated molecule substructures and ignores the target property when evaluating the similarity. Tailored similarity, on the other hand, uses only the descriptors that correlate with the target property. Therefore, while structural similarity produces more generic multi-purpose chemical spaces, tailored similarity produces property-sensitive focused chemical spaces. Figure 2 and Figure 3 show visualizations of the same datasets by using structural and tailored similarity methods, respectively. In ChemPlot, the similarity type can be assigned while creating the Plotter object. The default value for the similarity type is "tailored" when a target list is provided, otherwise, it is "structural". The following code shows the similarity type assignment.

$$
c p=\text { Plotter.from_smiles(smiles_list, target=target_list, sim_type="structural”) }
$$

\section{Plotting Options}

ChemPlot supports both static and interactive plotting formats. The static plots are images that can be exported in several file formats such as PNG, JPEG, PDF, or SVG. The visualizations shown in Figures 1-3 are examples of static plots. Differently, interactive plots allow users to drag, zoom, and highlight sections of the visualized data. Hovering over the data points displays a tooltip that contains the $2 \mathrm{D}$ chemical drawing and the target value of the molecule for that data point. Users have the option

(a) BBBP by PCA

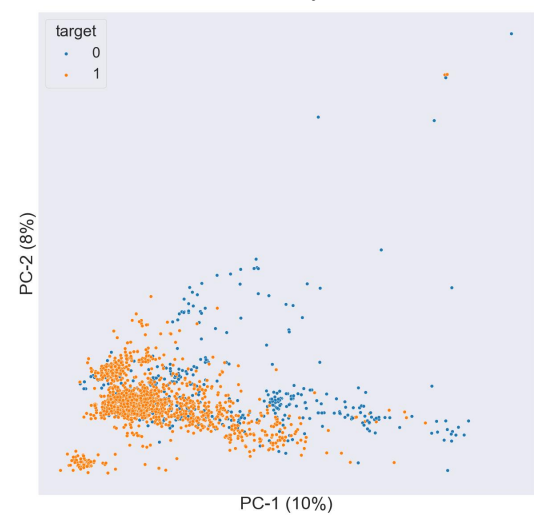

(d) AqSolDB by PCA

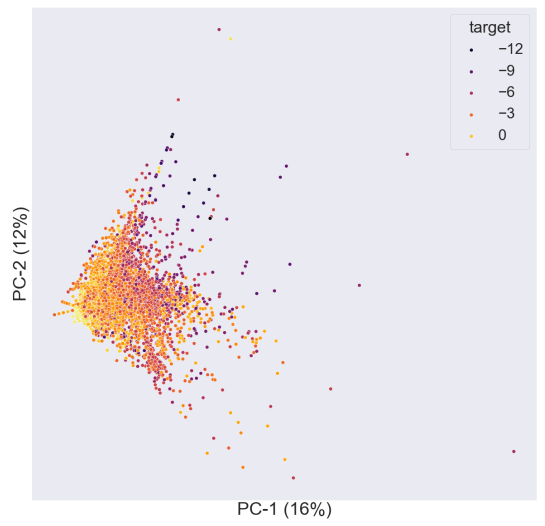

(b) BBBP by t-SNE

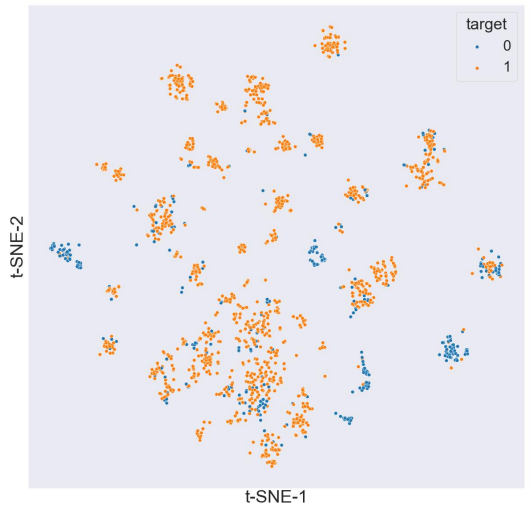

(e) AqSolDB by t-SNE

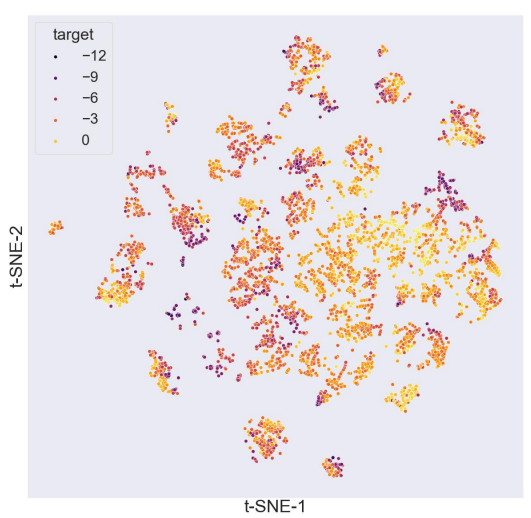

(c) BBBP by UMAP

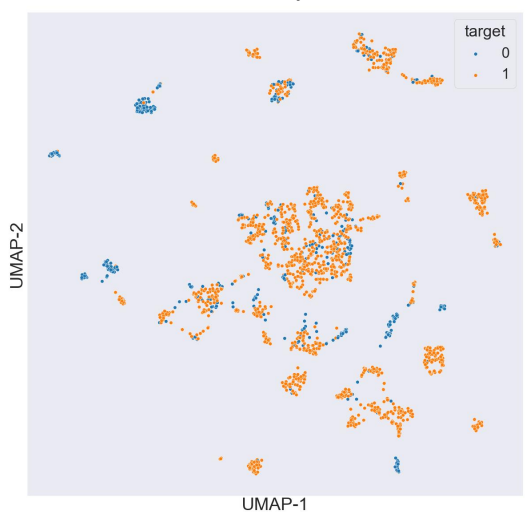

(f) AqSolDB by UMAP

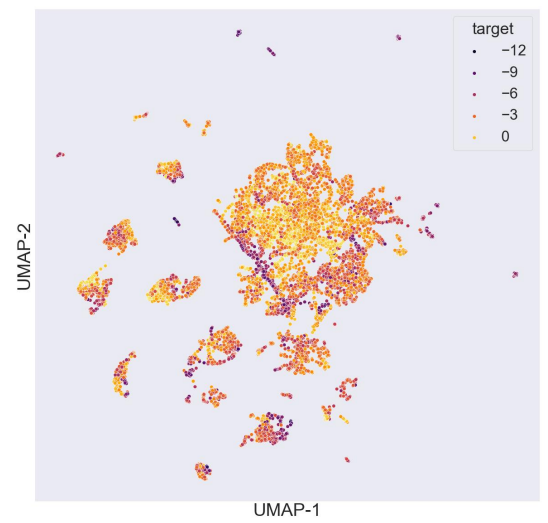

Figure 3. BBBP $(\mathbf{a}, \mathbf{b}$, and $\mathbf{c})$ and AqSolDB $(\mathbf{d}, \mathbf{e}$, and $\mathbf{f})$ datasets are visualized in the same layout as in Figure 2, but by using tailored similarity. Compared to the structural similarity, for the plots reduced by PCA, the explained variances are higher but the usage of the space is less efficient. For the plots reduced by t-SNE and UMAP, the clusters are separated from each other more clearly. 

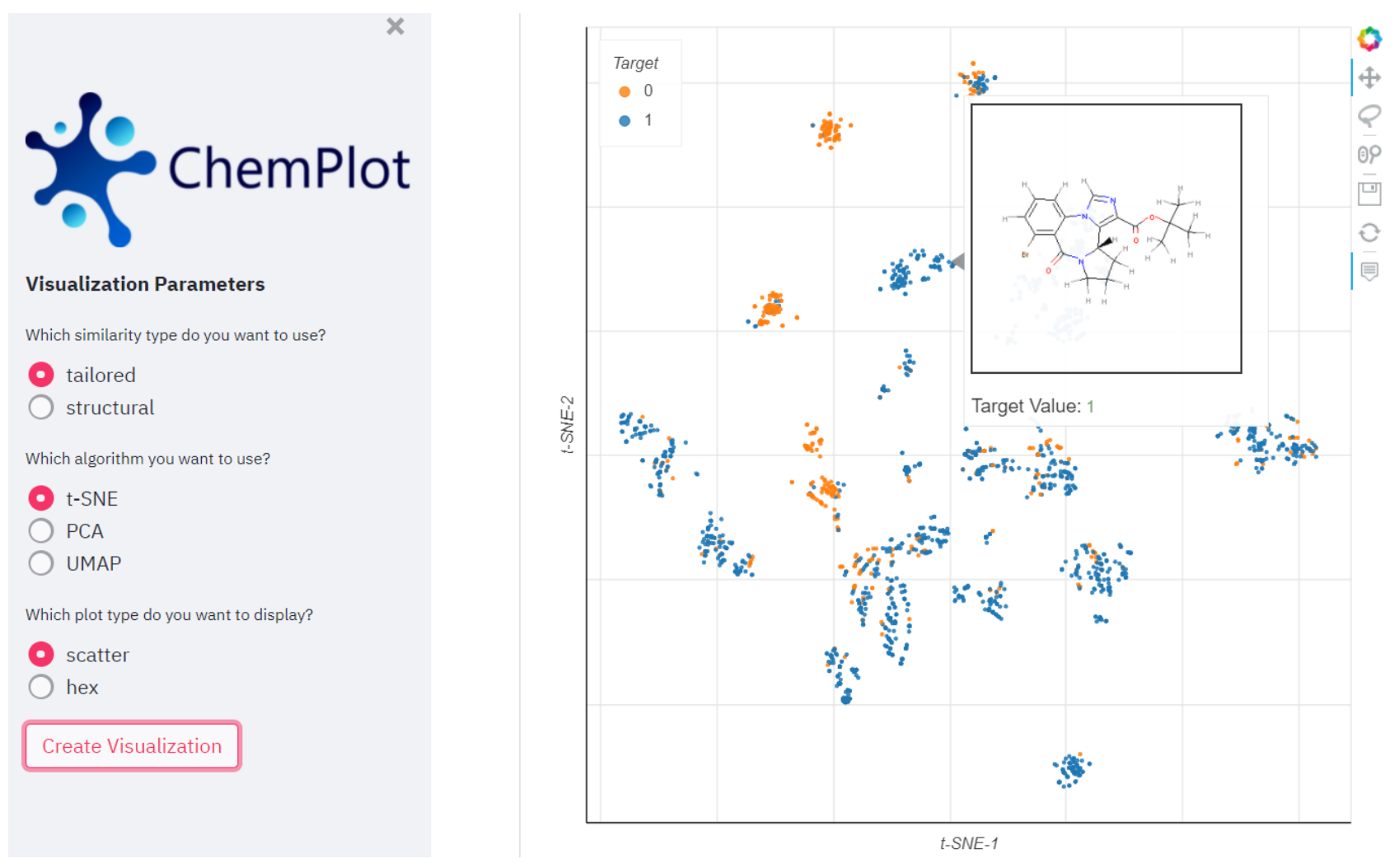

Figure 4. ChemPlot web application visualizing the interactive plot of the BBBP dataset. The left panel contains the configuration options. The right panel contains the interactive plot that is created according to the selected configuration. Hovering over the datapoints displays a tool-tip containing the 2D image of the molecule. From the top-right panel, users can activate the drag, highlight, zoom, save, and reset functions.

of saving the images of their plots both in their original and newly edited states. Furthermore, the interactive plot is created in HTML format, so that the users can export and embed it, for instance, to their own websites. Figure 4 shows an example interactive plot of the BBBP dataset. The following code shows the interactive plot generation of the chemical space as reduced by using PCA.

$$
\begin{aligned}
& c p \cdot p c a() \\
& c p \text {.interactive_plot }(\text { show_plot=True })
\end{aligned}
$$

\section{Additional Features}

ChemPlot contains stochastic processes (t-SNE and UMAP) that generate different visualizations for each run. In order to create reproducible plots, users can set the random state parameter as shown below.

$$
c p \text {. umap ( random_state }=0)
$$

ChemPlot allows users to remove outlier molecules. This option is controlled by a boolean parameter that's by default set to "False". To remove the outliers in data, users can set this parameter to "True" as shown below.

$$
\text { cp. visualize -plot (remove_outliers=True) }
$$


(a) AqSolDB (Scatter)

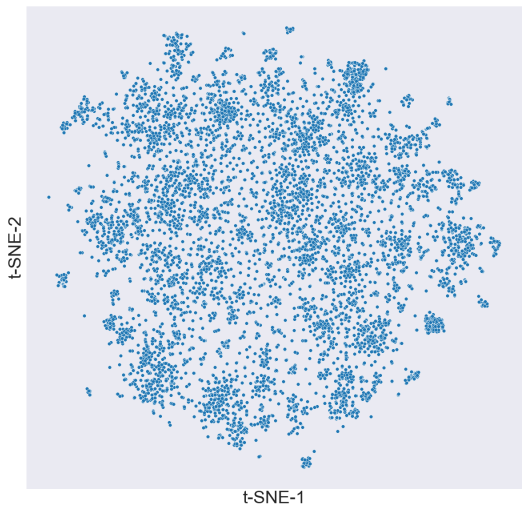

(b) AqSolDB (KDE)

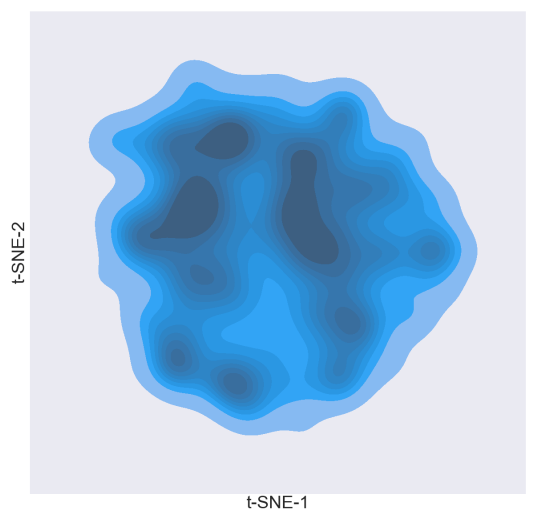

(c) AqSolDB (Hexagonal Bin)

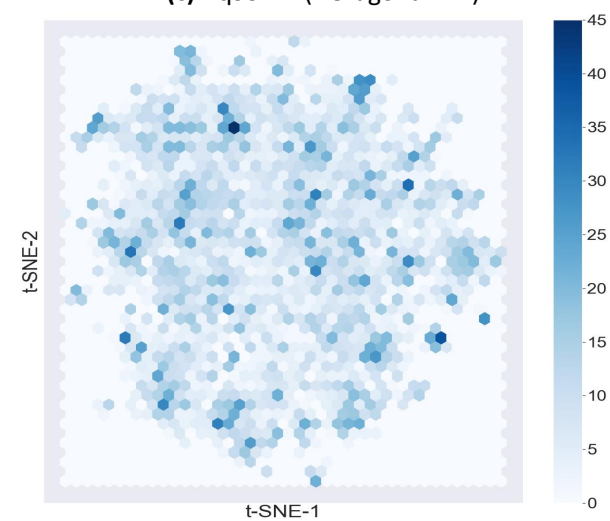

Figure 5. AqSolDB dataset visualized using t-SNE and structural similarity methods. The different sub-figures demonstrate the three plotting options of ChemPlot. Sub-figure a shows the scatter plot, where each point corresponds to a molecule.

Sub-figure $\mathbf{b}$ shows the kernel density estimation plot, where dark colors show high molecular density and light colors show low density in the corresponding area. Sub-figure c shows the hexagonal bin plot, where the colors of each hexagon indicate the density of molecules it contains. The color scale on the right shows the number of molecules found in the corresponding colors.

To speed up the plotting process for the large datasets, ChemPlot comes with a PCA pre-reduction option that reduces the number of dimensions to 10 before applying the t-SNE or UMAP dimensionality reduction methods. Users can set the parameter to "True" to apply PCA pre-reduction.

$$
\begin{aligned}
& \text { cp. umap }(P C A=\text { True }) \\
& c p \cdot \text { tsne }(P C A=\text { True })
\end{aligned}
$$

ChemPlot produces square images and the default size for static plots is $20 \times 20$ inches and for interactive plots is $700 \times 700$ pixels. Users can define custom sizes for both plotting options as shown below.

$$
\begin{aligned}
& c p \cdot \text { visualize }-p l o t(\operatorname{size}=20) \\
& c p . \text { interactive }-p l o t(\operatorname{size}=700)
\end{aligned}
$$

The default plotting type is Scatter in ChemPlot. In addition to the Scatter plot, ChemPlot provides Kernel Density Estimation (KDE) and Hexagonal plotting options. Figure 5 shows the visualization of the same dataset by the three plotting types. Users can set the plotting type as shown below.

$$
\begin{aligned}
& c p \cdot \text { visualize_plot }(\text { kind }=" k d e ") \\
& c p \cdot \text { visualize_plot }(\text { kind="hex" })
\end{aligned}
$$

\section{Software Testing}

Software testing is an indispensable step for robust and sustainable software development. For this purpose, we implemented three types of testing: i) unit tests, ii) visualization tests, and iii) performance tests.

Unit tests aim to isolate each individual unit of the software and validate whether they perform as expected or not. We 


\begin{tabular}{|c|c|c|c|c|c|c|c|c|}
\hline Dataset & Size & Target & Num. of Dim. & Similarity & Construction & PCA & t-SNE & UMAP \\
\hline FreeSolv $^{25}$ & 642 & numerical & 30 & tailored & 2.25 & 0.02 & 1.73 & 2.88 \\
\hline FreeSolv 25 & 642 & - & 991 & structural & 0.85 & 0.10 & 1.86 & 2.90 \\
\hline Huuskonen $^{26}$ & 1291 & numerical & 16 & tailored & 6.35 & 0.03 & 5.08 & 4.12 \\
\hline Huuskonen $^{26}$ & 1291 & - & 1684 & structural & 1.99 & 0.41 & 4.99 & 4.42 \\
\hline $\mathrm{BACE}^{21}$ & 1513 & numerical & 25 & tailored & 11.37 & 0.01 & 4.67 & 4.29 \\
\hline $\mathrm{BACE}^{21}$ & 1513 & - & 1690 & structural & 2.61 & 0.45 & 5.18 & 4.99 \\
\hline Lipophilicity $^{22}$ & 4200 & numerical & 17 & tailored & 27.31 & 0.04 & 16.42 & 18.09 \\
\hline Lipophilicity $^{22}$ & 4200 & - & 2035 & structural & 6.93 & 1.66 & 19.77 & 7.00 \\
\hline AqSolDB $^{24}$ & 9982 & numerical & 23 & tailored & 47.95 & 0.08 & 52.23 & 9.34 \\
\hline AqSolDB $^{24}$ & 9982 & - & 2046 & structural & 14.87 & 3.90 & 64.27 & 17.50 \\
\hline ClinTox $^{22}$ & 1478 & categorical & 69 & tailored & 9.82 & 0.04 & 4.67 & 4.23 \\
\hline ClinTox $^{22}$ & 1478 & - & 2007 & structural & 2.41 & 0.54 & 6.59 & 5.51 \\
\hline $\mathrm{BACE}^{21}$ & 1513 & categorical & 63 & tailored & 11.50 & 0.02 & 4.76 & 4.32 \\
\hline $\mathrm{BACE}^{21}$ & 1513 & - & 1690 & structural & 2.66 & 0.44 & 5.24 & 5.04 \\
\hline $\mathrm{BBBP}^{23}$ & 2039 & categorical & 69 & tailored & 12.34 & 0.03 & 6.84 & 5.93 \\
\hline $\mathrm{BBBP}^{23}$ & 2039 & - & 2016 & structural & 3.26 & 0.74 & 8.58 & 7.37 \\
\hline $\mathrm{HIV}^{22}$ & 41127 & categorical & 126 & tailored & 453.23 & 1.28 & 294.90 & 28.06 \\
\hline $\mathrm{HIV}^{22}$ & 41127 & - & 2048 & structural & 74.20 & 19.38 & 429.01 & 179.83 \\
\hline
\end{tabular}

Table 1. The performance results of ChemPlot for the various configurations on the benchmark datasets. The elapsed-time (s) is shown separately for the construction and reduction phases.

designed and implemented a total of 193 test cases that cover all combinations of parameters for all the available methods in ChemPlot. The current version of ChemPlot successfully passed all the unit tests.

Visualization tests aim to validate whether the visualization output is properly displayed. For this purpose, we developed an automated test code that creates a PDF file of plots, which are generated by using different parameters according to the given datasets of molecules. The produced PDF file is inspected and validated by the tester. The current version of ChemPlot is tested on three different molecular datasets ${ }^{21,23,26}$ and all the generated plots successfully passed the visual inspection tests.

Performance tests aim to evaluate the speed of the software under different circumstances. For this purpose, we developed an automated code to gauge the visualization performance of ChemPlot for the well-known molecular datasets ${ }^{21-26}$ of sizes between 642 and 41,127 molecules. The performance metrics of ChemPlot on an ordinary personal computer (processor with $2.80 \mathrm{GHz}$ clock speed and $8 \mathrm{~GB}$ of RAM) are shown in Table 1 . The data shows the elapsed-time (s) for the two sequential phases of the visualization process. The former is the construction phase, which shows the time spent for converting SMILES notations into numeric multi-dimensional representations. The latter is the reduction phase, which shows the time spent for reducing the multi-dimensions to 2D. We executed the reducing phase for the three reduction algorithms (PCA, t-SNE, and UMAP) separately. The construction phase of the structural similarity is linearly correlated with the size of the dataset and it is about three to six times faster than tailored similarity. For the largest dataset shown in Table 1, the construction time is approximately 74 and $453 \mathrm{~s}$ for structural and tailored similarities, respectively. In the reduction phase, while the elapsed-time for PCA is linearly correlated with the size of the dataset, it is polynomially correlated for UMAP and t-SNE. For the largest dataset, the reduction time using structural similarity was approximately 19, 180, and 429 s for PCA, UMAP, and t-SNE. 


\begin{tabular}{|c|c|c|c|c|c|c|c|c|c|c|c|c|c|c|c|c|c|c|}
\hline \multirow{3}{*}{ Dataset } & \multicolumn{6}{|c|}{ PCA } & \multicolumn{6}{|c|}{ t-SNE } & \multicolumn{6}{|c|}{ UMAP } \\
\hline & \multicolumn{2}{|c|}{$1 \%$} & \multicolumn{2}{|c|}{$2 \%$} & \multicolumn{2}{|c|}{$5 \%$} & \multicolumn{2}{|c|}{$1 \%$} & \multicolumn{2}{|c|}{$2 \%$} & \multicolumn{2}{|c|}{$5 \%$} & \multicolumn{2}{|c|}{$1 \%$} & \multicolumn{2}{|c|}{$2 \%$} & \multicolumn{2}{|c|}{$5 \%$} \\
\hline & $\mathbf{S}$ & $\mathbf{T}$ & $\mathbf{S}$ & $\mathbf{T}$ & $\mathbf{S}$ & $\mathbf{T}$ & $\mathbf{S}$ & $\mathbf{T}$ & $\mathbf{S}$ & $\mathbf{T}$ & $\mathbf{S}$ & $\mathbf{T}$ & $\mathbf{S}$ & $\mathbf{T}$ & $\mathbf{S}$ & $\mathbf{T}$ & $\mathbf{S}$ & $\mathbf{T}$ \\
\hline FreeSolv ${ }^{25}$ & 2.27 & 2.23 & 2.63 & 2.42 & 2.85 & 2.63 & 1.63 & 1.46 & 2.09 & 1.74 & 2.75 & 2.26 & 2.24 & 1.60 & 2.65 & 1.90 & 3.23 & 2.43 \\
\hline Huuskonen $^{26}$ & 1.77 & 1.10 & 1.81 & 1.18 & 1.86 & 1.31 & 1.39 & 0.97 & 1.62 & 1.10 & 1.84 & 1.27 & 1.52 & 1.00 & 1.65 & 1.15 & 1.86 & 1.33 \\
\hline $\mathrm{BACE}^{21}$ & 1.03 & 1.20 & 1.07 & 1.25 & 1.18 & 1.29 & 0.81 & 0.89 & 0.90 & 1.01 & 1.06 & 1.18 & 0.82 & 0.92 & 0.89 & 1.06 & 1.11 & 1.25 \\
\hline Lipophilicity $^{22}$ & 1.29 & 1.26 & 1.30 & 1.26 & 1.32 & 1.27 & 1.16 & 1.12 & 1.26 & 1.18 & 1.33 & 1.24 & 1.25 & 1.15 & 1.32 & 1.19 & 1.38 & 1.23 \\
\hline AqSolDB $^{24}$ & 2.24 & 1.91 & 2.25 & 1.95 & 2.28 & 2.01 & 2.11 & 1.69 & 2.25 & 1.85 & 2.39 & 1.98 & 2.12 & 1.80 & 2.19 & 1.93 & 2.30 & 2.08 \\
\hline ClinTox $^{22}$ & 0.11 & 0.11 & 0.11 & 0.12 & 0.11 & 0.12 & 0.10 & 0.11 & 0.12 & 0.12 & 0.13 & 0.12 & 0.13 & 0.12 & 0.14 & 0.13 & 0.15 & 0.12 \\
\hline $\mathrm{BBBP}^{23}$ & 0.23 & 0.22 & 0.23 & 0.22 & 0.23 & 0.23 & 0.17 & 0.19 & 0.20 & 0.21 & 0.24 & 0.26 & 0.20 & 0.19 & 0.22 & 0.22 & 0.24 & 0.25 \\
\hline
\end{tabular}

Table 2. Top 1, 2, and 5\% DPR scores of ChemPlot for the various configurations as experimented on the sample datasets.

\section{Distance Property Relationship Comparison}

In this set of experiments, we compare the performances of various configurations of chemical space visualization on dealing with the APC problem. While traditional methods like Structure-Activity Relationships (SAR) ${ }^{27}$ and Structure-Activity Landscape (SAL) ${ }^{28}$ indexes provide quantification and detection of APCs of molecular datasets, there is no available method for quantifying APCs for chemical space visualizations. To measure the activity/property difference of similar (i.e. close) molecules in the visualized chemical space, we propose a new metric, the Distance Property Relationship (DPR) scores. Unlike SAR and SAL indexes, the DPR score defines the similarity based on distances of molecules on the reduced chemical space as opposed to basing it on their common chemical substructures. To achieve this, the DPR algorithm first calculates the Euclidean distances between all pairs of molecules and sorts them in ascending order (i.e. closest first). Next, for a selected top percentage, an average of the property difference is calculated. Table 2 shows the top 1,2, and 5\% DPR scores of the chemical space visualizations using various experiment configurations. On all datasets, among the three dimensionality reduction methods, the t-SNE produced the best DPR scores, while UMAP performed better than PCA. Moreover, for the majority of experiments, the tailored similarity showed a better performance than structural similarity.

\section{Web Application}

The ChemPlot web application provides users with an easy-to-use application programming interface (API) for visualizing chemical spaces. Users can upload their molecular datasets and choose the available visualization options from the left panel. The API supports main functionalities of ChemPlot, including the similarity methods, dimensionality reduction methods, and plotting types. Additionally, it can be used to remove outliers and set a random state for reproducible plots. It generates interactive plots and allows users to export their visualized chemical spaces as an image or HTML file. Figure 4 shows an example view of the web application, which is openly accessible at https://www . amdlab.nl/chemplot/.

\section{DISCUSSION}

In this study, we introduced ChemPlot, a Python library for chemical space visualization. When designing ChemPlot, we aimed to provide a straightforward library where coding would be smooth and intuitive. This was achieved by providing a simple code flow and naming, handling the function parameters automatically, and preparing a descriptive user manual. ChemPlot allows users to convert molecular data files into chemical space visualizations simply by using a few lines of code and without setting 
any input parameters. Moreover, we developed a web application to streamline the visualization, such as for daily use and for users with no coding background. Thus, the ChemPlot Python library, alongside its user-friendly web application, is designed to serve a broad community of users.

A noteworthy feature of ChemPlot is the application of tailored similarity for the visualization of chemical spaces. Before discussing this similarity feature, it is important to understand the similarity concept. Similarity is relative and it depends on the target and the population, therefore there is no globally applicable definition of similarity ${ }^{11}$. The traditionally used, structural similarity attempts to evaluate the similarity using all possible aspects of the given molecular datasets and without taking the target property into account. This approach is very suitable when one wants to have a general overview of the dataset $^{29}$. However, structural similarity often fails when searching for new molecules with target properties. For example, when searching for molecules with a desired aqueous solubility value, one can expect that the neighboring molecules on the visualized chemical space would have close solubility values. Neighboring molecules, however, are more likely to have closer solubility values when, instead of the similarity defined by all the features, the similarity is defined essentially by the features that are known to affect the solubility. In relation to this, ChemPlot includes the tailored similarity option, which automatically identifies the correlated features with the given target and visualizes the chemical space based on them. The chemical spaces that are visualized by using the tailored similarity are less likely to be affected by the APC issue. Inline with this, for the majority of the datasets shown in Table 2, the tailored similarity produced lower DPR scores than the structural similarity.

In addition to a prominent use in high-throughput virtual screening, diversity analysis, and virtual library design, it is also possible to use ChemPlot for visualizing the applicability domain of AI models. Here, the applicability domain refers to the region of chemical space where a model is expected to perform reliable and make low-uncertainty predictions. By visualizing the applicability domain of the model, the level of confidence of the prediction for new molecules can be determined. For instance, when the predicted molecule is inside the applicability domain then the prediction can be considered as confident, and when it is outside the applicability domain then the prediction can be considered as uncertain. Moreover, by using ChemPlot it is possible to verify whether the dataset is properly split into train and test sets that adequately cover the chemical space ${ }^{30,31}$. Furthermore, a test set region that is not covered by the training set can be intentionally put aside in order to evaluate the extrapolation capability of the model ${ }^{31}$. Because of all these reasons, we expect that ChemPlot will serve as a complementary tool to the AI models that are frequently applied in molecular informatics studies.

Advancing research software sustainability is a dire challenge. Software released as desktop or web applications can suffer from a lack of maintenance, therefore over time, can become inaccessible or completely unusable. We released ChemPlot as an open-source library on GitHub, thereby enabling the community to contribute to its future development. Code developers may contribute, such as by adding new features pertaining to dimensionality reduction techniques or similarity methods. Additionally, they may have a chance to resolve user-specific technical issues when encountered. The integrity of the newly developed features can be validated by following the test procedures that are described in the Methods section. To pave the way 
for long-term software quality, we share the responsibility in maintenance and development of ChemPlot. Thus, it could evolve dynamically in parallel to the future needs of the community.

The most significant practical limitation of ChemPlot is the computation time that will be required for excessively large datasets. In our performance tests, we encountered bottlenecks in both the construction and the reduction phases. In the construction phase, the calculation time of fingerprints and descriptors depends on the total number of molecules and on the number of atoms in the molecules. Also, the tailored similarity feature requires an additional step of feature selection. ChemPlot uses the least absolute shrinkage and selection operator (lasso) regression analysis method and the logistic regression algorithms for feature selection. Depending on the algorithm used in this step, additional computation time is required. Therefore, the construction phase takes more time for the tailored similarity. To accelerate this process, the selection algorithms could be optimized or replaced entirely by faster methods for large datasets. In the reduction phase, the production of all the plots took less than three minutes for both PCA and UMAP methods. However, for t-SNE, the production of the plots of large datasets took significantly longer time. For instance, for the largest dataset we tested (41,127 instances), it took approximately seven minutes to generate the plot by t-SNE (see Table 1). We used Barnes-Hut ${ }^{32}$ implementation from scikit-learn $^{33}$ library which runs in $O(N \log N)$, where $N$ is the number of instances. The reduction time is naturally also affected by the number of dimensions that will be reduced. Therefore, it is usually recommended to use t-SNE once after the dimensions are reduced by PCA to a fixed value ${ }^{32,34}$ and meanwhile accepting a loss of information. Although ChemPlot has the PCA pre-reduction option, the performance tests shown in Table 1 were conducted without this pre-reduction step. Therefore, for t-SNE, the total time consumed is largely penalized by the number of dimensions as well. To increase t-SNE's processing rate, it may be worthwhile to implement its recently proposed variants ${ }^{34-37}$ in ChemPlot in future.

\section{METHODS}

\section{Structural Similarity}

When computing the chemical space visualization based on the structural similarity, the list of molecules is converted into ExtendedConnectivity Fingerprints (ECFPs) ${ }^{38}$. ECFPs are bit-vectors where each bit represents the presence or absence of a particular substructure. Substructures are extracted from the main structure by starting from each non-hydrogen atom and extending to the neighbor atoms until a specified distance is reached. Extracted substructures are hashed and mapped into fixed-sized bit-vector. ChemPlot uses the RDKit ${ }^{1}$ library to convert SMILES and InChI notations into ECFPs with a bit-vector length of 2,048 bits and radius of 2 adjacent atoms. After each molecule is converted, for all molecules, the bits that contain only 0 s or only $1 \mathrm{~s}$ are removed from the bit-vectors. The remaining number of bits determines the total number of dimensions and they are used as the input for the dimensionality reduction phase.

\section{Tailored Similarity}

When computing the chemical space visualization based on the tailored similarity, the list of molecules is converted into a set of descriptors as computed by using the Mordred library ${ }^{3}$. Initially, a total of 201 physico-chemical descriptors are calculated. A molecule is removed from the dataset in the case when a descriptor could not have been successfully computed. The list of descriptors is then used to form a matrix, in which the rows represent compounds and the columns represent descriptors. Next, the descriptors that are correlated with the target property 
are selected by using lasso regression for the numerical target values or by using logistic regression for the categorical target values. Lasso regression uses $L 1$ regularization with 0.05 of alpha(regularization multiplier) and has a maximum iteration of 10,000. Logistic regression uses $L 1$ regularization with 0.3 of $C$ (inverse of regularization strength) and liblinear as the optimization method. The resulting matrix of the selected descriptors is used as the input for the dimensionality reduction phase.

\section{Principal Component Analysis}

$\mathrm{PCA}^{4}$ is a linear dimensionality reduction algorithm, which projects the data points onto principal components by maximizing the variance. ChemPlot applies PCA from scikit-learn ${ }^{33}$ library with the default parameters. The two most significant principal components are used as the reduced dimensions (axes of the graph) in the visualization step.

\section{t-distributed Stochastic Neighbor Embedding}

$\mathrm{t}-\mathrm{SNE}^{7}$ is a non-linear dimensionality reduction algorithm that converts the similarities between data points into joint probabilities. It then minimizes the difference between the joint probability distributions of the high-dimensional data and the low-dimensional embedding. It is a stochastic process that produces different results from different initialization parameters. Except the perplexity parameter, ChemPlot applies $\mathrm{t}$-SNE from scikit-learn ${ }^{33}$ library using its default parameters. The perplexity parameter is computed automatically by the pre-trained model as described below.

\section{Uniform Manifold Approximation and Projection}

$\mathrm{UMAP}^{10}$ is a non-linear dimensionality reduction algorithm that constructs a particular weighted k-neighbor graph for the given data points and then computes a low dimensional layout of the graph. It is based on a stochastic process that produces different results from different initialization parameters. ChemPlot applies UMAP with the default parameters provided by the UMAP library, except the $n$ neighbors and min dist that are computed automatically by the pre-trained model described below.

\section{Automatic Parameter Assignment}

The most important parameters that affect the visualization are perplexity for t-SNE and number of neighbors for UMAP. However, it is not possible to set a fixed value that works best for all datasets. In order to determine the best parameter values for a given dataset, we developed a linear model. For this purpose, we used 20 molecular datasets of varying sizes and generated multiple plots for each dataset by assigning different values to the target parameters. The best plots for human perception and the respective values were selected by two users for each dataset based on the clearness and the balanced distribution of the clusters and data points. The selected values were used as the labels to form linear equations between the data size and the target parameters. By using the trained model, ChemPlot automatically assigns the parameters to generate visually meaningful plots.

\section{Static Plot}

ChemPlot uses the Seaborn ${ }^{39}$ library for generating the static plots. Static plots can be created by using one of three different visualization options, including the scatter plot, hexagonal bin plot, and kernel density plot. Static plots can be exported in PNG, JPEG, PDF, and SVG file formats. 


\section{Interactive Plot}

ChemPlot uses the Bokeh ${ }^{40}$ library for generating the interactive plots. As is clear from its name, the interactive plot allows users to interact actively with the visualization. Additionally, the interactive plot provides drag, highlight, zoom, save, and reset functions. Hovering over the data points displays a tool-tip that contains a basic image of the molecule as rendered by RDKit ${ }^{1}$. The interactive plot can be exported in HTML format.

\section{Removing Outliers}

ChemPlot identifies the molecules as outliers in the case when their $\mid z$-score $\mid \geq 3$. Z-scores are calculated by using SciPy ${ }^{41}$ library. The molecules that are identified as outliers can be removed from the plots by setting the remove outliers parameter to "True".

\section{Unit Tests}

Chemplot uses unittest Python framework for automated unit testing. Unit tests cover all construction, dimensionality reduction, and visualization methods. For all test cases, the related explanatory text is also provided. Unit tests are executed on three types of datasets: i) a dataset with a numerical target, ii) a dataset with a categorical target, and iii) a dataset that contains erroneous SMILES representations.

\section{Visualization Test}

Visualization test only covers the static plots. It generates plots for the selected datasets with all possible combinations of parameters and puts them into a single PDF file for human inspection. It does not contain any automated validation.

\section{Performance Test}

Performance test measures the elapsed-time for the construction and the dimension reduction phases. The test is executed for nine molecular datasets of different sizes and by using all the similarity and dimension reduction methods. Performance test provides two output files, where one includes a table of execution times shown in $\mathrm{s}$ and the other contains additional information on the test environment.

\section{Web Application}

ChemPlot web application is designed as an independent open-source software that imports ChemPlot as a library. It uses Streamlit ${ }^{42}$ library for creating the web interface.

\section{Code Availability}

The source code of ChemPlot and the web application is available on GitHub repositories, respectively (https://github.com/ mcsorkun/ChemPlot, https://github.com/mcsorkun/ChemPlot-web). The user manual is accessible via https:// chemplot.readthedocs.io/. The collective repositories of ChemPlot can be found at AMD group GitHub account on https: //github.com/ergroup/Chemplot.

\section{References}

1. Rdkit: Cheminformatics and machine learning software (2013).

2. Yap, C. W. Padel-descriptor: An open source software to calculate molecular descriptors and fingerprints. J. Comput. Chem. 32, 1466-1474 (2011). 
3. Moriwaki, H., Tian, Y.-S., Kawashita, N. \& Takagi, T. Mordred: a molecular descriptor calculator. J. Cheminformatics 10, 1-14 (2018).

4. Wold, S., Esbensen, K. \& Geladi, P. Principal component analysis. Chemom. Intell. Lab. Syst. 2, 37-52 (1987).

5. Kohonen, T. The self-organizing map. Proc. IEEE 78, 1464-1480 (1990).

6. Agrafiotis, D. K., Rassokhin, D. N. \& Lobanov, V. S. Multidimensional scaling and visualization of large molecular similarity tables. J. Comput. Chem. 22, 488-500 (2001).

7. Van der Maaten, L. \& Hinton, G. Visualizing data using t-sne. J. Mach. Learn. Res. 9 (2008).

8. Probst, D. \& Reymond, J.-L. Visualization of very large high-dimensional data sets as minimum spanning trees. J. Cheminformatics 12, $1-13(2020)$.

9. Maggiora, G. M. \& Bajorath, J. Chemical space networks: a powerful new paradigm for the description of chemical space. J. Comput. Mol. Des. 28, 795-802 (2014).

10. McInnes, L., Healy, J. \& Melville, J. Umap: Uniform manifold approximation and projection for dimension reduction. arXiv preprint https://arxiv.org/abs/1802.03426 (2018).

11. Maggiora, G., Vogt, M., Stumpfe, D. \& Bajorath, J. Molecular similarity in medicinal chemistry: miniperspective. J. Medicinal Chem. 57, 3186-3204 (2014).

12. Gütlein, M., Karwath, A. \& Kramer, S. Ches-mapper-chemical space mapping and visualization in 3 d. J. Cheminformatics 4, 1-16 (2012).

13. Sander, T., Freyss, J., von Korff, M. \& Rufener, C. Datawarrior: an open-source program for chemistry aware data visualization and analysis. J. Chem. Inf. Model. 55, 460-473 (2015).

14. Gonzalez-Medina, M. \& Medina-Franco, J. L. Platform for unified molecular analysis: Puma. J. Chem. Inf. Model. 57, 1735-1740 (2017).

15. Medina-Franco, J. L., Sánchez-Cruz, N., López-López, E. \& Díaz-Eufracio, B. I. Progress on open chemoinformatic tools for expanding and exploring the chemical space. J. Comput. Mol. Des. 1-14 (2021).

16. Takahashi, Y., Konji, M. \& Fujishima, S. Molspace: a computer desktop tool for visualization of massive molecular data. J. Mol. Graph. Model. 21, 333-339 (2003).

17. Škoda, P. \& Hoksza, D. Chemical space visualization using viframe. In 2013 IEEE/ACIS 12th International Conference on Computer and Information Science (ICIS), 541-546 (IEEE, 2013).

18. Awale, M., van Deursen, R. \& Reymond, J.-L. Mqn-mapplet: Visualization of chemical space with interactive maps of drugbank, chembl, pubchem, gdb-11, and gdb-13. J. Chem. Inf. Model. 53, 509-518 (2013).

19. Awale, M., Probst, D. \& Reymond, J.-L. Webmolcs: a web-based interface for visualizing molecules in three-dimensional chemical spaces. J. Chem. Inf. Model. 57, 643-649 (2017).

20. Gute, B. D., Basak, S. C., Mills, D. \& Hawkins, D. M. Tailored similarity spaces for the prediction of physicochemical properties. Internet Electron. J. Mol. Des 1, 374-387 (2002).

21. Subramanian, G., Ramsundar, B., Pande, V. \& Denny, R. A. Computational modeling of $\beta$-secretase 1 (bace-1) inhibitors using ligand based approaches. J. Chem. Inf. Model. 56, 1936-1949 (2016).

22. Wu, Z. et al. Moleculenet: a benchmark for molecular machine learning. Chem. Sci. 9, 513-530 (2018). 
23. Martins, I. F., Teixeira, A. L., Pinheiro, L. \& Falcao, A. O. A bayesian approach to in silico blood-brain barrier penetration modeling. J. Chem. Inf. Model. 52, 1686-1697 (2012).

24. Sorkun, M. C., Khetan, A. \& Er, S. Aqsoldb, a curated reference set of aqueous solubility and 2 descriptors for a diverse set of compounds. Sci. Data 6, 1-8 (2019).

25. Mobley, D. L. \& Guthrie, J. P. Freesolv: a database of experimental and calculated hydration free energies, with input files. J. Comput. Mol. Des. 28, 711-720 (2014).

26. Huuskonen, J. Estimation of aqueous solubility for a diverse set of organic compounds based on molecular topology. J. Chem. Inf. Comput. Sci. 40, 773-777 (2000).

27. Peltason, L. \& Bajorath, J. Sar index: quantifying the nature of structure- activity relationships. J. Medicinal Chem. 50, 5571-5578 (2007).

28. Guha, R. \& Van Drie, J. H. Structure- activity landscape index: identifying and quantifying activity cliffs. J. Chem. Inf. Model. 48, 646-658 (2008).

29. Sorkun, E., Zhang, Q., Khetan, A., Sorkun, M. C. \& Er, S. Reddb, a computational database of electroactive molecules for aqueous redox flow batteries. ChemRxiv preprint 10.26434/chemrxiv.14398067.v1 (2021).

30. Sorkun, M. C., Koelman, J. V. A. \& Er, S. Pushing the limits of solubility prediction via quality-oriented data selection. Iscience 24, 101961 (2021).

31. Redpred: Redox energy prediction tool for redox flow battery molecules. https://github.com/mcsorkun/RedPred/ (2021).

32. Van Der Maaten, L. Accelerating t-sne using tree-based algorithms. The J. Mach. Learn. Res. 15, 3221-3245 (2014).

33. Pedregosa, F. et al. Scikit-learn: Machine learning in Python. J. Mach. Learn. Res. 12, 2825-2830 (2011).

34. Linderman, G. C., Rachh, M., Hoskins, J. G., Steinerberger, S. \& Kluger, Y. Fast interpolation-based t-sne for improved visualization of single-cell rna-seq data. Nat. Methods 16, 243-245 (2019).

35. Ulyanov, D. Multicore-tsne. https://github.com/DmitryUlyanov/Multicore-TSNE (2016).

36. Chan, D. M., Rao, R., Huang, F. \& Canny, J. F. t-sne-cuda: Gpu-accelerated t-sne and its applications to modern data. In 2018 30th International Symposium on Computer Architecture and High Performance Computing (SBAC-PAD), 330-338 (IEEE, 2018).

37. Fujiwara, Y., Ida, Y., Kanai, S., Kumagai, A. \& Ueda, N. Fast similarity computation for t-sne. In 2021 IEEE 37 th International Conference on Data Engineering (ICDE), 1691-1702 (IEEE, 2021).

38. Rogers, D. \& Hahn, M. Extended-connectivity fingerprints. J. Chem. Inf. Model. 50, 742-754 (2010).

39. Waskom, M. L. seaborn: statistical data visualization. J. Open Source Softw. 6, 3021 (2021).

40. Bokeh Development Team. Bokeh: Python library for interactive visualization (2018).

41. Virtanen, P. et al. Scipy 1.0: fundamental algorithms for scientific computing in python. Nat. Methods 17, 261-272 (2020).

42. Streamlit (2021).

\section{Acknowledgements}

The authors acknowledge funding from the initiative "Computational Sciences for Energy Research" of Shell and the Dutch Research Council (NWO) grant no 15CSTT05. This work was sponsored by NWO Exact and Natural Sciences for the use of supercomputer facilities. 


\section{Author contributions}

M.C.S. and D.M. developed the codes for ChemPlot and its web application. S.E. supervised the project. All authors contributed to the writing of the manuscript.

\section{Competing interests}

The authors declare no competing interests. 